Canadian Oncology

Nursing Journal

Revue canadienne

de soins infirmiers

en oncologie

Volume 29, Issue 3 • Summer 2019

elSSN: 2368-8076 


\section{PERSPECTIVES INTERNATIONALES}

\section{Cancer du col utérin : connaissances des étudiantes de premier cycle d'un collège sélectionné de Lalitpur, au Népal}

\author{
par Rachana K.C. et Rita Giri
}

\section{RÉSUMÉ}

Le cancer du col utérin est le deuxième cancer en importance chez les femmes des pays en développement. Au Népal, on en sait peu sur létat des connaissances des jeunes femmes adultes à propos de ce cancer. Une étude transversale descriptive a donc été réalisée auprès d'étudiantes de premier cycle afin de déterminer ce qu'elles savent. Un questionnaire autoadministré a permis de recueillir des données auprès d'un échantillon non probabiliste de 150 étudiantes du collège de gestion Little Angels à Lalitpur, au Népal. Ces données ont ensuite fait lobjet d'une analyse statistique descriptive et déductive.

L'âge moyen des répondantes était de 19,3 \pm 1,1 an. Le niveau de connaissances sur le cancer du col utérin s'est révelé inadéquat chez presque toutes les participantes (95\%). Par ailleurs, $56 \%$ d'entre elles savaient ce qu'est le cancer du col utérin, et $35 \%$ en connaissaient moyennement les facteurs de risque. Près du deux tiers des étudiantes savaient qu'on peut prévenir ce cancer. Parmi les mesures préventives, $68,5 \%$ des participantes ont parlé d'avoir une bonne hygiène, 38,3\% de recevoir un vaccin, 19,5\% d'utiliser le condom et 8,7\% de faire un test Pap. Seulement 11,3\% des répondantes ont indiqué déjà connaître le vaccin contre le VPH. Aucune association statistique significative entre les connaissances et les variables $d u$ groupe à létude (âge, religion, origine ethnique, revenu familial, statut tabagique et pratiques sexuelles) n'a pu être établie.

Les résultats permettent de conclure que les étudiantes ont un niveau de connaissances inadéquat à propos $d u$ cancer $d u$ col utérin. Ces résultats reflètent bien la nécessité des campagnes

\section{AU SUJET DES AUTEURES}

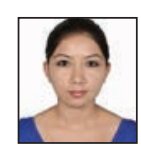

Rachana K.C. détient un baccalauréat en sciences infirmières de I'Université Tribhuvan. Elle est infirmière en chef à l'hôpital Norvic, au Népal.

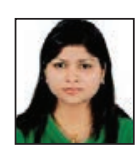

Rita Giri est titulaire d'une maîtrise en sciences infirmières de I'Université Tribhuvan. Elle travaille en tant qu'infirmière dans différents centres hospitaliers du Népal et a été chargée de cours et superviseure clinique dans différentes écoles de soins infirmiers du Népal.

Auteure-ressource : Rita Giri

Courriel : ritagiri2044@gmail.com

Téléphone : 1-778-995-9629

DOI:10.5737/23688076293189193 destinées à sensibiliser les étudiants et le public en général au sujet de ce cancer (symptômes, causes, facteurs de risque, mesures de prévention, etc.).

\section{INTRODUCTION}

À l'échelle mondiale, le cancer du col utérin est le quaAtrième cancer le plus répandu chez les femmes. On estime à 530000 le nombre de nouveaux cas en 2012, et ce cancer est responsable de 7,5\% de tous les décès dus au cancer, chez la femme. Dans les pays en développement, le cancer du col utérin est le deuxième cancer en importance : approximativement 44500 nouveaux cas y ont été diagnostiqués en 2012, soit $84 \%$ des nouveaux cas recensés partout dans le monde. En 2012, environ 270000 femmes ont succombé au cancer du col utérin; plus de $85 \%$ de ces décès sont survenus dans des pays à faible revenu ou à revenu intermédiaire (OMS, 2016). Aux États-Unis, l'incidence du cancer du col utérin et la mortalité qui y est associée ont diminué de façon importante au cours des 40 dernières années grâce aux tests Pap (Statistiques sur le cancer du col utérin, Centers for Disease Control and Prevention [CDC]).

Au Népal, le cancer du col utérin serait la principale cause de décès liés au cancer. Le risque de développer la maladie est donc grand pour les 10,16 millions de Népalaises âgées de 15 ans et plus. Le nombre annuel de nouveaux cas de cancer du col utérin est de 2332 , tandis que le nombre de décès attribuables chaque année à ce type de cancer s'élève à I 367 (Information Centre on HPV and Cancer, 2016). L'infection par le virus du papillome humain est la principale cause de cancer du col utérin, les autres facteurs de risque étant les prédispositions génétiques, le faible statut socioéconomique, l'activité sexuelle précoce (avant 17 ans), les partenaires sexuels multiples, l'immunosuppression et le tabagisme (Chintamani, 2011). La prévention primaire du cancer du col utérin comprend la vaccination contre le virus du papillome humain (VPH) des filles âgées de 9 à 13 ans, afin de les protéger avant qu'elles ne deviennent actives sexuellement. La prévention secondaire se fait, chez les femmes de plus de 30 ans, au moyen d'outils de dépistage tels que l'IVA (inspection visuelle après application d'acide acétique), le test Pap ou le test de dépistage du VPH, suivi d'un traitement des lésions précancéreuses détectées pouvant entraîner un cancer du col utérin (OMS, 2014).

Même s'il s'agit d'une maladie tout à fait évitable, les 
Népalaises, contrairement aux habitantes des pays occidentaux, ne sont pas en mesure de prévenir le cancer du col utérin en raison de leur manque de connaissances concernant la vaccination, laquelle n'est par ailleurs pas toujours offerte ou accessible à cause des coûts. La prévention primaire, comme la détection précoce par une sensibilisation accrue, l'utilisation de vaccins prophylactiques anti-VPH chez les jeunes adolescentes de 9 à 13 ans avant qu'elles soient exposées à l'infection, les programmes de dépistage structurés, le diagnostic et le traitement jouent un rôle majeur dans la diminution de la morbidité et de la mortalité liées au cancer du col utérin (Sherpa et al., 2015; Dangal, 2012).

À l'heure actuelle, on en sait très peu sur le niveau de connaissances des jeunes femmes au sujet du cancer du col utérin et de ses facteurs de risque. Pourtant, ces dernières courent un risque réel de contracter une infection au VPH. C'est dans cette optique qu'a été sélectionné l'échantillon de cette étude, composé d'étudiantes qui appartiennent à une tranche d'âge admissible au vaccin anti-VPH et constituent un groupe où le risque d'être infectée par le VPH est élevé. Les résultats de cette étude, qui explore leurs connaissances sur divers aspects du cancer du col utérin, pourraient réellement aider à identifier les lacunes et à concevoir des messages éducatifs ciblés et efficaces.

\section{MÉTHODOLOGIE}

Une étude transversale descriptive a été réalisée afin de mesurer l'étendue des connaissances sur le cancer du col utérin de jeunes femmes étudiant au collège de gestion Little Angels de Lalitpur, au Népal. Léchantillon, composé à l'aide d'une méthode non probabiliste, regroupait 150 étudiantes de premier cycle. Les données ont été recueillies grâce à un questionnaire autoadministré structuré fondé sur des études antérieures. En tout, 21 questions de type « correct/incorrect » ont été élaborées afin de mesurer le niveau de connaissances; des points étaient attribués pour chaque bonne réponse. Le questionnaire recueillait des renseignements sur les caractéristiques sociodémographiques, les connaissances sur le cancer du col utérin et les pratiques sexuelles. Le niveau de connaissances était établi selon le barème suivant : Inadéquat - Moins de $50 \%$ de bonnes réponses; Moyen - 50 à $74 \%$ de bonnes réponses; Adéquat $-75 \%$ et plus de bonnes réponses.

Les données ont été recueillies au cours d'une période de 10 jours, au mois d'octobre 2016. Des questionnaires papier ont été distribués individuellement dans la salle de classe, en veillant à respecter l'anonymat. La participation à l'étude était volontaire et le consentement éclairé écrit des répondantes a été obtenu avant leur participation. Le Nepal Health Research Council a validé le caractère éthique de l'étude.

Les données ont été saisies et analysées au moyen de la version 23 du Statistical Package for Social Sciences (SPSS). Le questionnaire comptait 10 questions évaluant les connaissances sur le cancer du col utérin; un point étant accordé pour chaque bonne réponse. Une analyse de la variance (ANOVA) a été réalisée pour tester les associations entre les variables nominales. Le niveau de signification statistique était fixé à 0,05.

\section{RÉSULTATS}

Le tableau 1 montre que la plupart (95,3\%) des répondantes avaient un niveau de connaissances inadéquat sur le cancer du cancer du col utérin.

Parmi les participantes, $56 \%$ ont répondu correctement aux questions portant sur la compréhension de la maladie. De même, la moitié (50,7 \%) d'entre elles savaient que le fait d'avoir plusieurs partenaires sexuels augmente le risque de développer un cancer du col utérin. En moyenne, $35 \%$ des répondantes présentaient un niveau de connaissances adéquat des facteurs de risque de la maladie.

Les répondantes devaient indiquer, parmi les 5 options, quels étaient les signes et symptômes du cancer du col utérin. Le tableau 3 montre que la réponse la plus fréquente $(68,2 \%)$ a été « écoulements vaginaux inhabituels », suivi de « saignements très abondants pendant les menstruations » $(28,4 \%)$, puis de « saignements entre les menstruations » $(26,4 \%)$. Une minorité des répondantes $(14,9 \%)$ ont identifié les saignements après les relations sexuelles comme un symptôme de la maladie.

\begin{tabular}{|l|c|c|}
\hline \multicolumn{3}{|l|}{ Tableau 1. Niveau de connaissances sur le cancer du col utérin } \\
\hline Variables & Fréquence & Pourcentage \\
\hline Inadéquat & 143 & 95,3 \\
\hline Moyen & 7 & 4,7 \\
\hline Moyenne \pm É.-T. & $31,42 \pm 11,07$ & Étendue (9-72,7) \\
\hline
\end{tabular}

\begin{tabular}{|c|c|c|}
\hline Caractéristiques & Fréquence & Pourcentage \\
\hline \multicolumn{3}{|l|}{ Compréhension } \\
\hline Lésion du col utérin & 40 & 26,7 \\
\hline Douleur au col utérin & 4 & 2,7 \\
\hline $\begin{array}{l}\text { Croissance anormale des } \\
\text { cellules au col utérin }\end{array}$ & 84 & 56 \\
\hline Gonflement du col utérin & 22 & 14,7 \\
\hline \multicolumn{3}{|l|}{ Facteurs de risque ${ }^{*}$} \\
\hline Tabagisme & 40 & 27 \\
\hline Infection au VPH & 57 & 38,5 \\
\hline Partenaires sexuels multiples & 75 & 50,7 \\
\hline Mariage précoce & 72 & 48,6 \\
\hline Obésité & 16 & 10,8 \\
\hline $\begin{array}{l}\text { Connaissances moyennes des } \\
\text { facteurs de risque } \pm \text { É. }-T \text {. }\end{array}$ & $\begin{array}{l}(35,1351 \pm \\
15,40768)\end{array}$ & $\begin{array}{l}\text { Étendue } \\
(20-100)\end{array}$ \\
\hline
\end{tabular}


Le tableau 4 montre que plus de la moitié (63,3\%) des répondantes ont indiqué que le cancer du col utérin peut être évité. La majorité des jeunes femmes interrogées (68,5\%) savaient que la prévention repose sur une bonne hygiène, tandis que seulement un peu plus de $40 \%$ d'entre elles connaissaient le dépistage par prélèvement de fluides et d'un échantillon de cellules au col utérin. Globalement, $30 \%$ des répondantes avaient un niveau de connaissances moyen des mesures préventives.

Tableau 3. Connaissances des signes et symptômes du cancer du col utérin $(n=150)$

\begin{tabular}{|l|c|c|}
\hline Caractéristiques & Fréquence & Pourcentage \\
\hline Signes et symptômes* & 21 & 14,2 \\
\hline Fièvre & 39 & 26,4 \\
\hline $\begin{array}{l}\text { Saignements entre les } \\
\text { menstruations }\end{array}$ & 101 & 68,2 \\
\hline $\begin{array}{l}\text { Écoulements vaginaux inhabituels } \\
\text { Saignements très abondants } \\
\text { pendant les menstruations }\end{array}$ & 42 & 28,4 \\
\hline $\begin{array}{l}\text { Saignements après les rapports } \\
\text { sexuels }\end{array}$ & 22 & 14,9 \\
\hline *Réponses multiples & & \\
\hline
\end{tabular}

\begin{tabular}{|c|c|c|}
\hline Caractéristiques & Fréquence & Pourcentage \\
\hline \multicolumn{3}{|l|}{$\begin{array}{l}\text { Possibilité de prévention du } \\
\text { cancer du col utérin }\end{array}$} \\
\hline Oui & 95 & 63,3 \\
\hline Non & 55 & 36,7 \\
\hline \multicolumn{3}{|l|}{ Mesures préventives* } \\
\hline Utilisation du condom & 29 & 19,5 \\
\hline Bonne hygiène & 102 & 68,5 \\
\hline Vaccin anti-VPH & 57 & 38,3 \\
\hline Test Pap & 13 & 8,7 \\
\hline \multicolumn{3}{|l|}{ Dépistage* } \\
\hline Biopsie du col utérin & 39 & 26,0 \\
\hline Prélèvement sanguin & 76 & 50,7 \\
\hline $\begin{array}{l}\text { Prélèvement de fluides/ } \\
\text { cellules au col utérin }\end{array}$ & 63 & 42,0 \\
\hline Analyse d'urine & 42 & 28,0 \\
\hline $\begin{array}{l}\text { Mesures préventives Moyenne } \\
\pm \text { É.-T. }(30,0671 \pm 12,65427)\end{array}$ & (étendue $=20-80$ ) & \\
\hline
\end{tabular}

Comme le montre le tableau 5, la majorité (88,7\%) des répondantes ne connaissaient pas le vaccin anti-VPH, et la majeure partie du groupe $(92,6 \%)$ pensait que le vaccin était réservé aux femmes. De plus, 34,8 \% des répondantes pensaient que le vaccin n'était offert que moyennant des frais dans un hôpital privé, et bon nombre $(44,7 \%$ ) des répondantes ont avoué ne pas savoir l'âge recommandé pour recevoir le vaccin. Le tableau 6 montre que très peu (4\%) des répondantes étaient actives sexuellement.

Le tableau 7 ne révèle aucune relation statistique significative entre les connaissances sur le cancer du col utérin et les variables déterminées (intervalle de confiance [IC] de $95 \%$ ).

\begin{tabular}{|c|c|c|}
\hline \multicolumn{3}{|c|}{ Tableau 5. Connaissances sur le vaccin anti-VPH $(n=150)$} \\
\hline Variables & Fréquence & Pourcentage \\
\hline \multicolumn{3}{|l|}{ Vaccin anti-VPH déjà connu } \\
\hline Oui & 17 & 11,3 \\
\hline Non & 133 & 88,7 \\
\hline \multicolumn{3}{|l|}{ Admissibilité au vaccin* } \\
\hline Sexe masculin & 33 & 22,1 \\
\hline Sexe féminin & 138 & 92,6 \\
\hline \multicolumn{3}{|l|}{ Disponibilité du vaccin* } \\
\hline $\begin{array}{l}\text { Moyennant des frais dans un } \\
\text { hôpital public }\end{array}$ & 10 & 15,2 \\
\hline $\begin{array}{l}\text { Moyennant des frais dans un } \\
\text { hôpital privé }\end{array}$ & 23 & 34,8 \\
\hline $\begin{array}{l}\text { Offert gratuitement dans } \\
\text { le cadre d'une campagne } \\
\text { gouvernementale }\end{array}$ & 22 & 33,3 \\
\hline Non disponible au Népal & 15 & 22,7 \\
\hline \multicolumn{3}{|l|}{ Âge recommandé pour le vaccin } \\
\hline Aucune réponse & 28 & 18,7 \\
\hline De 9 à 25 ans & 19 & 12,7 \\
\hline De 26 à 40 ans & 33 & 22,0 \\
\hline De 41 à 50 ans & 3 & 2,0 \\
\hline Je ne sais pas & 67 & 44,7 \\
\hline
\end{tabular}

\begin{tabular}{|l|c|c|}
\hline \multicolumn{3}{|c|}{ Tableau 6. Réponses concernant l'activité sexuelle $(n=150)$} \\
\hline Caractéristiques & Fréquence & Pourcentage \\
\hline Pratique sexuelle active & & \\
\hline Oui & 6 & 4,0 \\
\hline Non & 144 & 96,0 \\
\hline
\end{tabular}




\begin{tabular}{|c|c|c|c|c|c|}
\hline Variables & Fréquence & Moyenne & Écart-type & Valeur de F & Valeur de $P$ \\
\hline \multicolumn{6}{|l|}{ Âge } \\
\hline Inférieur ou égal à 20 & 130 & 31,1538 & 11,19303 & \multirow[t]{2}{*}{0,58} & \multirow[t]{2}{*}{0,44} \\
\hline 21 et plus & 20 & 33,1818 & 10,33371 & & \\
\hline \multicolumn{6}{|l|}{ Origine ethnique } \\
\hline Brahmane/Chhetri & 54 & 30,3872 & 11,77469 & \multirow[t]{5}{*}{1,02} & \multirow[t]{5}{*}{0,39} \\
\hline Janajati & 67 & 31,8860 & 11,20391 & & \\
\hline Madhesi & 23 & 30,4348 & 8,92775 & & \\
\hline Musulman & 2 & 36,3636 & 0,00000 & & \\
\hline Dalit & 4 & 40,9091 & 11,13404 & & \\
\hline \multicolumn{6}{|l|}{ Religion } \\
\hline Hindouisme & 115 & 31,3834 & 10,80315 & \multirow[t]{4}{*}{0,33} & \multirow[t]{4}{*}{0,80} \\
\hline Bouddhisme & 30 & 30,9091 & 12,76751 & & \\
\hline Islam & 2 & 38,6364 & 3,21412 & & \\
\hline Christianisme & 3 & 33,3333 & 6,94330 & & \\
\hline \multicolumn{6}{|l|}{ Revenu familial } \\
\hline$<10000$ & 9 & 27,7778 & 10,27626 & \multirow[t]{3}{*}{0,67} & \multirow[t]{3}{*}{0,51} \\
\hline De 10000 à 50000 & 92 & 31,2747 & 9,85414 & & \\
\hline$>50000$ & 49 & 32,3748 & 13,24023 & & \\
\hline \multicolumn{6}{|l|}{ Antécédents familiaux } \\
\hline Oui & 6 & 34,0909 & 14,86857 & \multirow[t]{2}{*}{0,36} & \multirow[t]{2}{*}{0,54} \\
\hline Non & 144 & 31,3131 & 10,93913 & & \\
\hline \multicolumn{6}{|l|}{ Tabagisme } \\
\hline Oui & 11 & 33,0579 & 15,35945 & \multirow[t]{2}{*}{0,25} & \multirow[t]{2}{*}{0,61} \\
\hline Non & 139 & 31,2950 & 10,72416 & & \\
\hline \multicolumn{6}{|l|}{ Pratique sexuelle $(n=6)$} \\
\hline Oui & 6 & 37,1212 & 12,66761 & \multirow[t]{2}{*}{1,66} & \multirow[t]{2}{*}{0,19} \\
\hline Non & 144 & 31,1869 & 10,98526 & & \\
\hline Statistiquement significatif & de variance ( & ir de $P<0,0$ & & & \\
\hline
\end{tabular}

\section{DISCUSSION}

Cette étude se limite au cas des étudiantes de premier cycle du collège de gestion de Lalitpur, au Népal. Les conclusions de l'étude ont mis en lumière le faible niveau de connaissances des répondantes sur le cancer du col utérin, et cela malgré qu'il s'agisse du cancer le plus meurtrier chez les femmes vivant au Népal. Les résultats obtenus ici sont similaires à ceux des études menées en Arabie Saoudite et en Inde où, respectivement, $98 \%$ et $95,7 \%$ des participantes connaissaient peu le cancer du col utérin (Al-Sheikh et al., 2014; Saha et al., 2010).
On constate également que plus de la moitié des répondantes ont répondu correctement aux questions portant sur la compréhension de la maladie, un résultat similaire à celui obtenu en Australie (Rao, Heathcote, Jackson et Rousseau, 2015).

De même, seule une minorité de répondantes ont identifié le test Pap comme une mesure préventive. Ce résultat s'apparente à ceux d'une étude réalisée en Inde, où seulement II \% des personnes interrogées connaissaient le test Pap. Cela prouve bien qu'il faut intensifier les programmes de sensibilisation à la santé (Al-Sheikh et al., 2014). 
La majorité des répondantes de la présente étude ne connaissaient pas le vaccin anti-VPH. Ce constat est le même que ceux rapportés par deux études réalisées à Johannesburg (Afrique du Sud) et à Kuala Lumpur où, respectivement, $88,2 \%$ et $73,7 \%$ des personnes interrogées ne savaient pas qu'il existait un vaccin visant à prévenir le cancer du col utérin (Rashwan, Ishak et Sawalludin, 2013; Kalau, 2012).

La présente étude n’a révélé aucune relation entre les connaissances sur le cancer du col utérin et l'âge. En Inde, le niveau de connaissances était associé à l'âge : les étudiantes de plus de 20 ans avaient un niveau de connaissances supérieur à celles qui avaient moins de 20 ans (Saha et al., 2010). Dans le même ordre d'idées, la présente étude ne permet pas non plus de faire de lien entre le revenu familial et le niveau de connaissances, des résultats qui diffèrent de ceux de l'étude réalisée à Johannesburg, en Afrique du Sud, où le niveau de connaissances était plus élevé chez les étudiants jouissant d'un statut socio-économique élevé, et plus faible chez les étudiants dont le revenu était plus faible (Rashwan, Ishak et Sawalludin, 2013).

\section{RÉFÉRENCES}

Al-Sheikh, G.K., Abnussaed, E.M., Fayed, A.A., Khan, F.H., Syed, S.B., Al-Taminni, T.N. et Elmorshedy, H.N. (2014). Knowledge of Saudi female university students regarding cervical cancer and acceptance of the Human papilloma virus vaccine. Saudi Medical Journal, 35(10), 1223-1230. doi:10.15537/smj.2015.2.11250

Centers for Disease Control. (2016, June 20). Centre for disease control and prevention. [en ligne] http://www.cdc.gov/CANCER/cervical/ statistics/

Chintamani (2011). Lewis' Medical and Surgical Nursing: Assessment and Management of Clinical Problems (7th edition). Elsevier: India Pvt ltd.

Dangal, G. (2012). Cervical Cancer and HPV Vaccine in Nepal: Issues and challenges. Nepal Journal of Obstetrics and Gynaecology, 6(2). doi:10.3126/njog.v6i2.6761

ICO Information Centre on HPV and Cancer (2016). Human Papillomavirus and related diseases report Nepal. Institut Catalàd'Oncologia [en ligne] http://www.hpvcentre.net/statistics/ reports/NPL.pdf

Kalau, E.K. (2012). Knowledge about Human papillomavirus, Human papillomavirus vaccine and cervical cancer among female students at the University of Witwatersrand and their sexual practice. [en ligne] http://wiredspace.wits.ac.za/bitstream/handle/ı0539/ı2530/ Final\%2oMmed\% 20PDF.pdf

\section{CONCLUSION}

Dans la présente étude, la plupart des étudiantes de premier cycle avaient un niveau de connaissances inadéquat sur le cancer du col utérin. Ce sont en fait les connaissances sur les mesures préventives et le vaccin anti-VPH qui se sont révélées le plus lacunaires chez les étudiantes. Par conséquent, des efforts devraient être faits au Népal pour sensibiliser davantage le public au cancer du col utérin et à ses mesures de prévention, particulièrement au test Pap et au vaccin contre le VPH, afin de prévenir la maladie.

\section{REMERCIEMENTS}

Les auteures aimeraient transmettre l'expression de leur plus sincère gratitude à la Janamaitri Foundation Institute of Health Sciences/LACHS affiliée à l'Institute of Medicine de l'Université Tribhuvan, qui ont approuvé la tenue de cette étude. L'équipe de recherche tient à remercier le collège de gestion Little Angels pour leur autorisation, leur aide et leur coopération pendant la collecte de données ainsi que toutes les participantes de l'étude.

Organisation mondiale de la santé (2014). WHO / New WHO guide to prevent and control cervical cancer. [en ligne] http://www.who.int/ mediacentre/news/releases/20I4/preventing-cervical-cancer/en/

Organisation mondiale de la santé (2016). WHO I Human papillomavirus (HPV) and cervical cancer. [en ligne] http://www. who.int/mediacentre/factsheets/fs380/en/

Rao, A., Heathcote, C., Jackson, M. et Rousseau, I. (2015). What is Cervical Cancer? - Cancer Council Victoria. [en ligne] http://www. cancervic.org.au/about-cancer/cancer_types/cervical_cancer

Rashwan, H., Ishak, I. et Sawalludin, N. (2013). Knowledge and views of secondary school students in Kuala Lumpur on cervical cancer and its prevention. Asian Pacific Journal of Cancer Prevention, 14. 10:7314/apjcp.2013.14.4.2545

Saha, A., Chaudhary, A.N., Bhowmik, P. et Chatterjee, R. (2010). Awareness of cervical cancer among female students of Premier Colleges in Kolkata, India. Asian Pacific Journal of Cancer Prevention, 11, 1085-1089. [en ligne] https://www.ncbi.nlm.nih.gov/ pubmed/21133629

Sherpa, A.T., Karki, B.S., Sundby, J., Nygard, M., Franceschii, S. et Clifford, G. (2015). Population based study of cervical cancer screening in Bharatpur, Nepal. J. Manmohan Memorial Inst. Health. Sci, 1(4). doi:10.3126/jmmihs.v1i4.11994 